\title{
Vacuolar and plasma membrane stripping and autophagic elimination of Toxoplasma gondii in primed effector macrophages
}

\author{
Yun M. Ling, ${ }^{1}$ Michael H. Shaw, ${ }^{1}$ Carol Ayala,${ }^{2}$ Isabelle Coppens, ${ }^{3}$ \\ Gregory A. Taylor, ${ }^{4}$ David J.P. Ferguson, ${ }^{5}$ and George S. Yap ${ }^{1}$ \\ 'Department of Molecular Microbiology and Immunology, Division of Biology and Medicine, Brown University, \\ Providence, RI 02912 \\ ${ }^{2}$ Core Research Laboratories, Rhode Island Hospital, Providence, RI 02903 \\ 3Department of Molecular Microbiology and Immunology, Johns Hopkins University School of Public Health, \\ Baltimore, MD 21205 \\ ${ }^{4}$ Department of Medicine, Department of Molecular Genetics \& Microbiology, Department of Immunology, \\ and Center for the Study of Aging, Duke University and GRECC VA Medical Center, Durham, NC 27710 \\ ${ }^{5}$ Nuffield Department of Pathology, John Radcliffe Hospital, Oxford University, Oxford OX3 9DU, UK
}

\begin{abstract}
Apicomplexan protozoan pathogens avoid destruction and establish a replicative niche within host cells by forming a nonfusogenic parasitophorous vacuole (PV). Here we present evidence for lysosome-mediated degradation of Toxoplasma gondii after invasion of macrophages activated in vivo. Pathogen elimination was dependent on the interferon $\gamma$ inducible-p47 GTPase, IGTP, required PI3K activity, and was preceded by PV membrane indentation, vesiculation, disruption, and, surprisingly, stripping of the parasite plasma membrane. Denuded parasites were enveloped in autophagosome-like vacuoles, which ultimately fused with lysosomes. These observations outline a series of mechanisms used by effector cells to redirect the fate of a classically nonfusogenic intracellular pathogen toward a path of immune elimination.
\end{abstract}

CORRESPONDENCE

George S. Yap:

George_Yap@brown.edu
Protozoan parasites of phylum Apicomplexa (for example, Plasmodium, Toxoplasma, Eimeria, and Cryptosporidium) represent important human and veterinary pathogens worldwide (1, 2). Apicomplexans actively invade host cells and form parasitophorous vacuoles (PVs), which appear to be secluded from the endocytic and lysosomal compartments of the infected cell $(3,4)$. Nevertheless, the PV appears to provide an interface through which the parasite acquires nutrients from the host cytosol for growth and replication and alters innate immune signaling $(5,6)$. The formation of a nonfusogenic PV has been classically viewed as an adaptive mechanism for intracellular survival by apicomplexan parasites, allowing parasite evasion of lysosomal destruction. The immune response to several apicomplexans is characterized by the development of Th1 responses, including the secretion of IFN- $\gamma$ and TNF- $\alpha$. In the case of Toxoplasma gondii infection, cell-autonomous IFN- $\gamma$ signaling of hemopoietic and

$\overline{\text { The online version of this article contains supplemental material. }}$ nonhemopoietic cells is critical for immunity (7). Although IFN- $\boldsymbol{\gamma}$-induced mechanisms (e.g., NO generation by type II NOS [8] and tryptophan degradation by IDO [9]) restricting parasite growth have been described, whether cytokine-activated cells can directly destroy Toxoplasma tachyzoites remains uncertain. In this study, we investigated the fate of tachyzoites invading in vivo-primed macrophages. Our in-depth analysis revealed a novel and sophisticated series of intracellular mechanisms used by macrophage effector cells to destroy nonfusogenic intracellular parasites.

\section{RESULTS AND DISCUSSION}

We designed an experimental system using mice primed with a uracil-auxotrophic carbamoyl phosphate synthase null (CPS) strain (Fig. S1 A, available at http://www.jem.org/cgi/ content/full/jem.20061318/DC1; reference 10) that allowed us to trace the fate of GFP-labeled T. gondii after entry into IFN- $\boldsymbol{\gamma}$-activated host cells in vivo. At day 7 after priming, when peritoneal cells express the IFN- $\boldsymbol{\gamma}$-inducible GTPase 

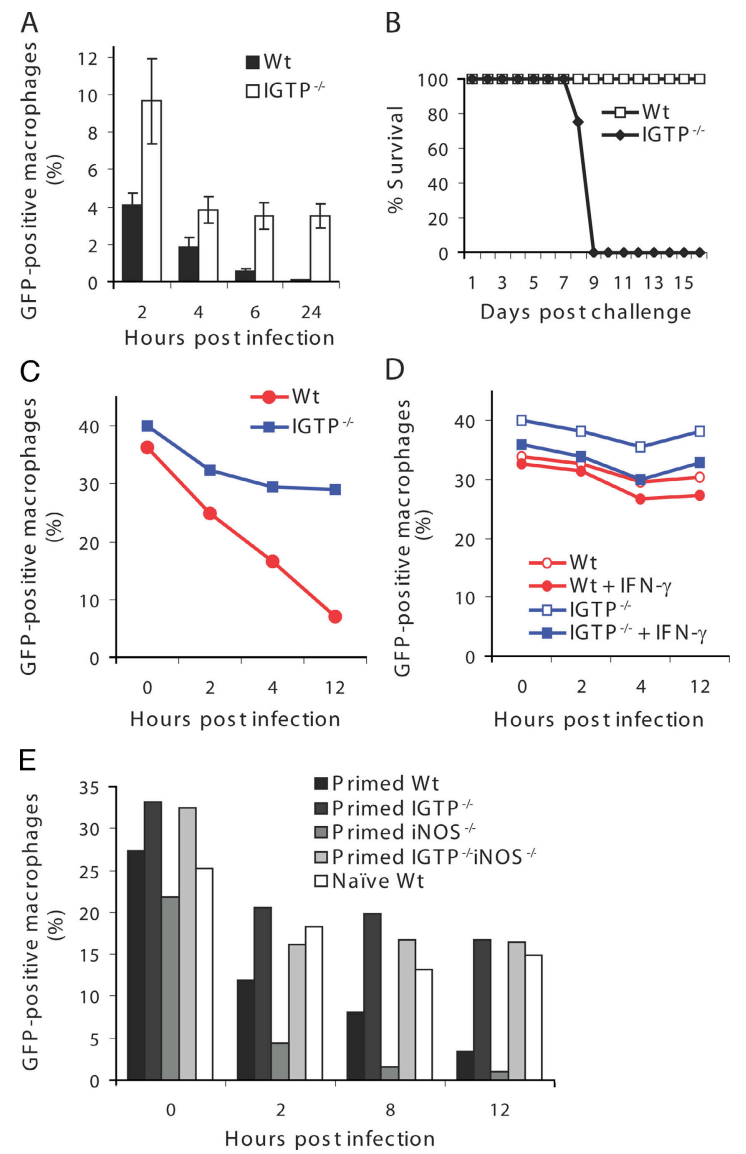

Figure 1. IGTP-dependent rapid T. gondii elimination in primed macrophages. (A) Impaired T. gondii elimination in primed IGTP-I- mice. CPS-primed (day 7 after infection) WT and IGTP-I- mice were challenged with $4 \times 10^{6}$ GFP-PTG i.p. Infection rates (mean \pm SEM) in peritoneal macrophages were determined by FACS $(n=3)$. (B) Survival of primed WT $(n=6)$ and IGTP $-1-(n=4)$ mice after GFP-PTG challenge. (C) Infection rates in primed WT and IGTP-I- peritoneal macrophages after GFPPTG infection ( $\mathrm{MOI}$ of $1,15 \mathrm{~min}$ ) ex vivo. Data shown are representative of eight experiments. (D) Thioglycolate-elicited WT and IGTP-I- peritoneal macrophages failed to eliminate T. gondii after GFP-PTG infection ( $\mathrm{MOI}$ of $1,15 \mathrm{~min}$ ) when activated in vitro. IFN- $\gamma$ treatment $(1 \mathrm{ng} / \mathrm{ml}$ ) started $12 \mathrm{~h}$ before infection. (E) IGTP-dependent rapid T. gondii elimination in primed macrophages does not require iNOS. Primed (WT, IGTP ${ }^{-1-}$, iNOS $^{-1-}$, and IGTP-I-iNOS ${ }^{-1-}$ ) and naive WT (thioglycolate-elicited) PECs were infected with GFP-PTG ex vivo. Data are representative of three experiments.

IGTP (Fig. S1 B), we challenged the mice i.p. with GFPPTG, a low-virulence type II strain of T. gondii, and monitored infection rate in peritoneal macrophages (large, granular cells that are mostly $\left.\mathrm{CD} 11 \mathrm{~b}^{+} \mathrm{F} 4 / 80^{+}\right)$, considered the primary effector cells against T. gondii (11). Infection in primed WT macrophages decreased rapidly, and by $24 \mathrm{~h}$ after challenge, few infected cells could be detected (Fig. 1 A). In mice, acute resistance to T. gondii infection is IGTP dependent (12). We therefore tested the ability of primed IGTP-deficient $\left(\mathrm{IGTP}^{-/-}\right)$mice to clear GFP-PTG. As shown in Fig. 1 A, the decrease in infection rate in $\mathrm{IGTP}^{-/-}$mice was highly attenuated, resulting in death of GFP-PTG-challenged $\mathrm{IGTP}^{-/-}$mice despite priming (Fig. $1 \mathrm{~B}$ ).

To avoid limitations of the in vivo priming challenge model, we infected primed peritoneal exudate cells (PECs) ex vivo. Primed WT macrophages still cleared GFP-PTG efficiently ex vivo, whereas $\mathrm{IGTP}^{-/-}$cells failed to do so (Fig. $1 \mathrm{C})$. The decrease of GFP positivity determined by flow cytometry reflected a reduction of infection rate observed directly by fluorescence microscopy and correlated with decreased plaque-forming efficiency (Fig. S2, available at http://www.jem.org/cgi/content/full/jem.20061318/DC1), indicating that primed macrophages eliminate rather than simply inhibit $T$. gondii. The microbicidal activity of primed macrophages is clearly IGTP dependent, confirmed by data (Fig. S3) showing that IGTP acts cell autonomously to effect parasite clearance. However, IGTP induction alone is not sufficient for parasite elimination given that IGTP-expressing thioglycollate-elicited macrophages pretreated with IFN- $\gamma$ in vitro were ineffectual (Fig. $1 \mathrm{D})$, suggesting that in vivo priming involves additional undefined accessory signals.

Earlier in vitro studies of the anti-Toxoplasma activity of macrophages indicated a critical role of inducible nitric oxide synthase (iNOS; reference 8). Nevertheless, iNOS-deficient (iNOS ${ }^{-/-}$) mice were capable of resisting acute $T$. gondii infection (13). To clarify the role of iNOS in the IGTP-dependent parasite elimination, we included primed $\mathrm{iNOS}^{-/-}$and $\mathrm{IGTP}^{-/-} \mathrm{iNOS}^{-/-}$cells in the experiment shown in Fig. $1 \mathrm{E}$. $\mathrm{iNOS}^{-/-}$macrophages cleared the parasites as efficiently as WT cells, if not better, indicating that iNOS is not required for the IGTP-dependent parasite elimination. We can also exclude antibody-mediated phagocytosis, as $\mu \mathrm{Mt}$ mice also cleared the parasite efficiently (Fig. S4, available at http:// www.jem.org/cgi/content/full/jem.20061318/DC1).

The nonfusogenic nature of the $T$. gondii $\mathrm{PV}$ was revealed using macrophages or other cell types without prior immune activation (4, 14). Given that primed macrophages rapidly eliminate invaded GFP-PTG, we wondered whether T. gondii can fuse with lysosomes in this system. We probed adherent PECs from primed mice infected with GFP-PTG in vivo for LAMP1. At $4 \mathrm{~h}$ after infection, a larger percentage of GFP-PTG colocalized with LAMP1 staining in primed WT than in primed IGTP $^{-/-}$cells (Fig. 2, A and B, and Videos $\mathrm{S} 1$ and $\mathrm{S} 2$, which are available at http://www.jem.org/cgi/ content/full/jem.20061318/DC1). LAMP1-associated parasites appeared to have weakened GFP intensity, probably a result of acidification-induced fluorescence quenching (15). Using a second method to label lysosomes, adherent PECs were pulsed with Texas red-labeled dextran before GFPPTG infection ex vivo. We consistently observed higher rates of fusion between Texas red-labeled lysosomes and GFP-PTG in primed WT macrophages than in $\mathrm{IGTP}^{-/-}$ cells (Fig. 2, C and D, and Fig. S5). To formally demonstrate the role of lysosomal fusion in parasite elimination, we used bafilomycin A1 to inhibit lysosomal acidification. Bafilomycin A1 treatment attenuated the parasite elimination in 

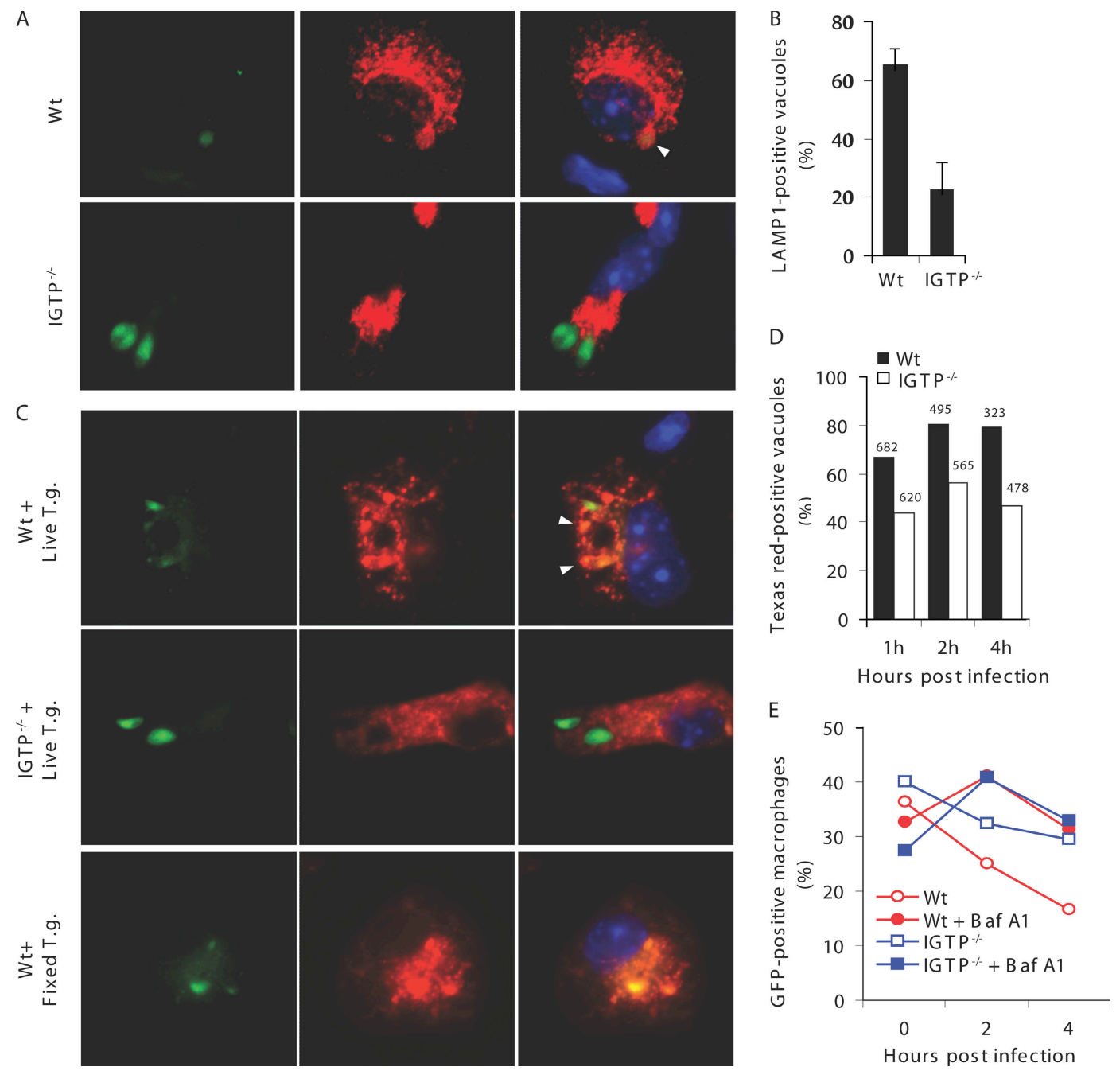

Figure 2. Evidence for lysosomal fusion with $T$. gondii in primed macrophages. (A) T. gondii reside in LAMP1 ${ }^{+}$compartments in primed macrophages. Primed mice were challenged with $4 \times 10^{6}$ GFP-PTG i.p. Adherent PECs were stained for LAMP1. Green, GFP-PTG; red, LAMP1; blue, DAPI. In A and $\mathrm{C}$, arrow heads point to lysosome-associated T. gondii with decreased GFP fluorescence. (B) Quantification of fusion events in the experiment depicted in A. Percentage of LAMP1 ${ }^{+} T$. gondii vacuoles (mean \pm SEM) was determined by counting 20-30 T. gondii-containing

primed WT macrophages (Fig. 2 E), suggesting a role for lysosomal fusion in parasite attrition.

To visualize intracellular events leading to $T$. gondii destruction, we examined the fine structures of the parasites and associated host membranes by electron microscopy (EM). We pulsed naive and primed macrophages with BSA-gold $(6 \mathrm{~nm})$ and chased for $3 \mathrm{~h}$ to label lysosomes before a $15-\mathrm{min}$ pulse infection. $15 \mathrm{~nm}$ BSA-gold was present during the infection to distinguish between actively invaded and phagocytosed parasites. Samples were prepared for EM analysis at 1, 2 , and $4 \mathrm{~h}$ after infection. Consistent with a nonphagocytic mode of entry, shortly after infection, the majority of the parasites in both primed WT and $\mathrm{IGTP}^{-/-}$macrophages vacuoles per sample ( $n=3$ mice per group). (C) T. gondii fuse with dextran-labeled lysosomes in primed macrophages. Green, GFP-PTG; red, dextran; blue, DAPI. (D) Quantification of fusion events in experiments depicted in C. Percentage of Texas red-positive T. gondii vacuoles was shown (the number above the bar shows the total number of T. gondiicontaining vacuoles counted). (E) Bafilomycin A1 (Baf A1) treatment $(100 \mathrm{nM})$ attenuated rapid parasite elimination in primed WT macrophages. Data are representative of three experiments.

were found within $15 \mathrm{~nm}$ of gold-negative PVs associated with host ER and mitochondria. We observed a markedly higher frequency of parasite degeneration in primed WT macrophages than in primed IGTP $^{-/-}$and naive cells (Fig. S6, A-C, available at http://www.jem.org/cgi/content/full/ jem.20061318/DC1). Although heterogeneity in the EM profiles within each sample was noted, contrasting features of parasite-host cell interface were clearly observed between primed WT and IGTP ${ }^{-/-}$macrophages. After careful analysis of distinct aberrations in the parasite vacuolar structure observed in primed WT macrophages, here we propose a series of events involving PV membrane (PVM) indentation, $\mathrm{PVM}$ vesiculation/disruption, and parasite plasma membrane 


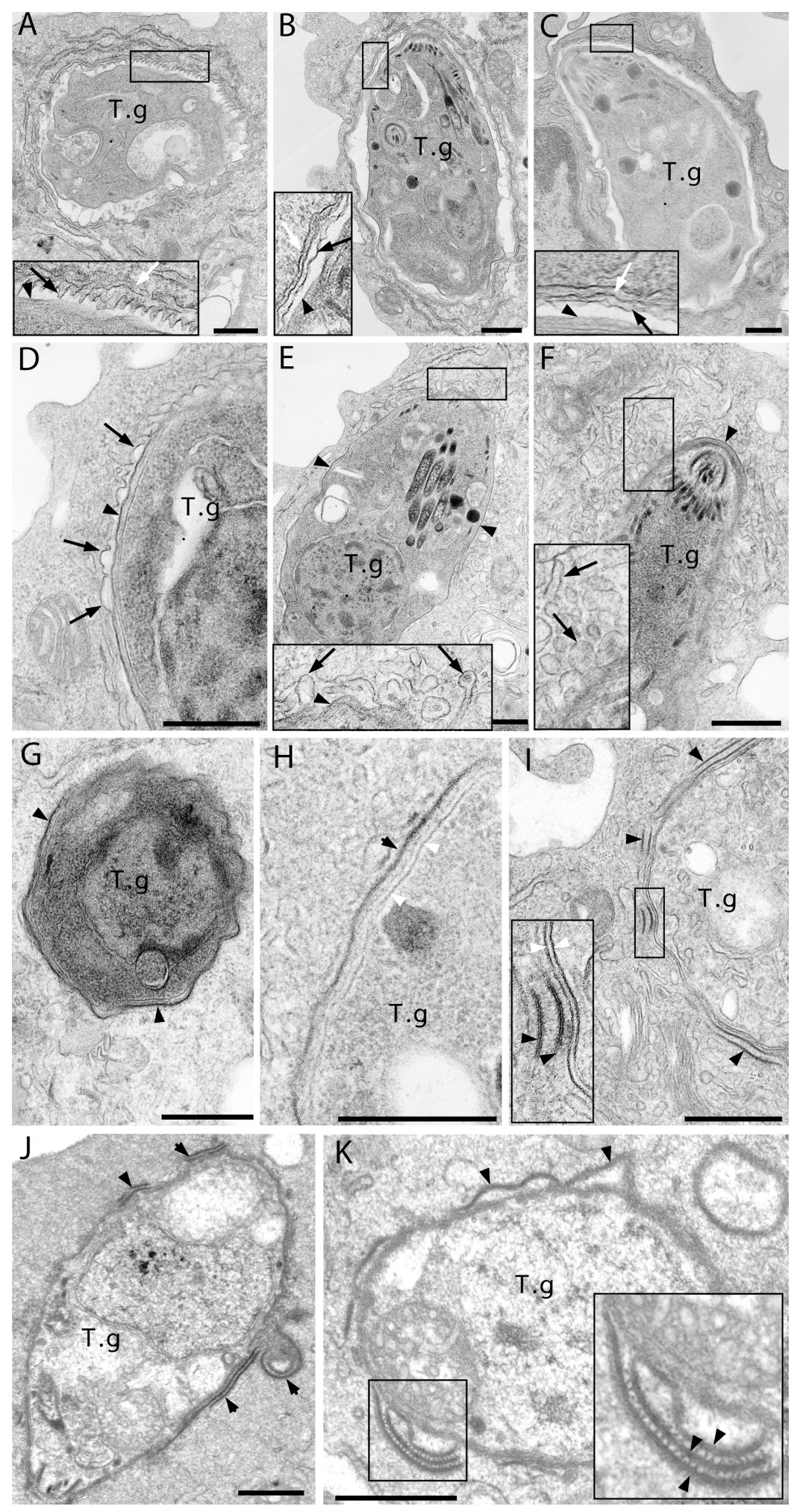

Figure 3. Ultrastructural alterations of T. gondii PVM and plasma membrane in primed WT macrophages. (A) T. gondii PVM indentation (phase I) in a primed WT macrophage. (B) Spacious PV in a primed IGTP-Imacrophage. (C) Normal PV in a thioglycolate-elicited peritoneal macrophage. (D-K) Images of T. gondii in primed WT macrophages. (D-F) PVM vesiculation and disruption (phase II). Irregular PVM forms cuplike projec- tions in D. ER is no longer associated with PVM undergoing vesiculation in E. PVM disintegrates into vesicles and tubules radiating away from the parasite in $\mathrm{F}$. $(\mathrm{G}$ and $\mathrm{H})$ Naked parasites in direct contact with host cytoso after PVM dissolution. Note electron-dense T. gondii plasmalemma in $\mathrm{H}$. (I and J) Parasite membrane stripping (phase III). Note that the electrondense plasmalemma has been severed, exposing the Inner membrane 
stripping (phase I, II, and III, respectively), which ultimately lead to lysosomal degradation (phase IV) of the parasite.

Phase I is characterized by the formation of tight-fitting parasite vacuoles in primed WT macrophages (Fig. $3 \mathrm{~A}$ ), unlike the normally spacious $T$. gondii vacuoles observed in primed $\mathrm{IGTP}^{-/-}$(Fig. $3 \mathrm{~B}$ ) and naive cells (Fig. $3 \mathrm{C}$ ). Tachyzoites in phase I PVs appeared intact, but little tubulovesicular network formation was observed in the PV lumen. PVMs in these tight vacuoles presented an undulating and corrugated profile, with inward protuberances measuring 23-60 $\mathrm{nm}$ in height, and seemed to closely contact the surface of the parasite more than $10-70 \%$ of the circumference of the PV (Fig. $3 \mathrm{~A}$ and Fig. S7, which is available at http:// www.jem.org/cgi/content/full/jem.20061318/DC1). PVM indentation toward the parasite may allow for pathogen recognition that initiates signals leading to subsequent PVM remodeling. Indeed, we obtained multiple images showing various degrees of PVM vesiculation, but only in primed WT macrophages. In earlier stages of phase II, the previously corrugated PVM presented a jagged and irregular profile, with subsequent formation of cuplike outward projections of the PVM (Fig. 3 D). Initiation of the vesiculation process was associated with a dispersal of the ER that typically underlies the PVM (Fig. 3 E). At later stages of phase II, when extensive vesiculation was observed, the PVM disintegrated into vesicles and elongated tubules radiating away from the parasite (Fig. 3 F). PVM dissolution resulted in the appearance of naked parasites in direct contact with the host cytosol (Fig. 3 $\mathrm{G})$. At this stage, the parasite membrane seemed to be intact, with a continuous electron-dense plasmalemma and the characteristic double-layered inner cell membrane complex (Fig. $3 \mathrm{H}$ ). In phase III, detachment of the parasite plasmalemma was evident (Fig. 3, I-K). Even more surprisingly, we obtained pictures clearly indicating that the parasite outer membrane has been severed and folded (Fig. 3, I and J). It appeared that the coated plasmalemma stripped from the parasite can form more complex stacks held together by electron-dense bridging structures (Fig. $3 \mathrm{~K}$ and Fig. S8).

Phase I, II, and III profiles in primed WT macrophages were frequent at 1 and $2 \mathrm{~h}$ after infection, and less so at $4 \mathrm{~h}$, indicating that PVM disruption and parasite denudation happens early and proceeds rapidly. Overall, the above events were rarely observed in naive macrophages and primed IGTP ${ }^{-/-}$cells. Of more than 100 T. gondii sections examined in primed IGTP $^{-/-}$macrophages, we found only one phase III-denuded parasite (vs. 15-20\% in primed WT cells), suggesting that IGTP could play an active role in the denudation process. However, the machinery that mediates plasmalemma severing and stripping in phase III remains enigmatic.
Our ultrastructural analysis of the fate of $T$. gondii within primed macrophages provides a clear picture of how an activated macrophage aggressively disrupts the intracellular niche, which the parasite attempts but fails to establish. In a study of $T$. gondii infection of in vitro IFN- $\boldsymbol{\gamma}$-activated astrocytes, Martens et al. (16) recently reported similar vesiculation and disruption of the PVM. However, how the parasite was eventually eliminated remained unclear because, unlike our results obtained in primed macrophages, no evidence for lysosomal fusion with $T$. gondii was observed in astrocytes.

We considered autophagy as the likely mechanism leading to the lysosomal destruction of the denuded parasites during phase IV. Although autophagy is normally considered a homeostatic cellular response to stress and starvation (17), recent reports have documented an emerging link between autophagy and antimicrobial defense (18-20). To probe the involvement of autophagy, we used inhibitors of PI3K function, which is required for autophagosome formation (21). As shown in Fig. $4 \mathrm{~A}$, the addition of three distinct PI3K inhibitors (wortmannin, LY294002, and 3-methyladenine) uniformly attenuated IGTP-dependent parasite elimination in primed effector cells, suggesting the involvement of an autophagy-like response against the parasite. Autophagic vacuoles are characteristically double membrane structures, which retain the fluorescent dye, monodansylcadaverine (MDC; reference 22). Indeed, we found extensive colocalization of GFP-PTG with MDC in primed WT macrophages and to a lesser extent in primed IGTP ${ }^{-/-}$cells (Fig. 4 B). Definitive evidence indicating autophagosome formation is provided by the EM images shown in Fig. 4 (C and D) and Fig. S9 (A-C), which is available at http://www.jem.org/cgi/content/full/ jem.20061318/DC1. Multiple autophagosome-like vacuoles surrounding entire parasites together with associated host cytosolic material were found in primed WT macrophages. Autophagosomes contained only parasites with disrupted plasma membrane, suggesting that leakage of internal antigens of the parasite may provide signals for autophagy induction. Fusion of flattened elongated vesicles appeared to give rise to double membrane structures characteristic of autophagosomes (Fig. 4 E; reference 23), which can ultimately fuse with lysosomes. Indeed, consistent with Fig. 2, we observed fusion of BSA-gold-labeled lysosomes with Toxoplasma-containing autophagic vacuoles (Fig. 4, F and G, and Fig. S10), indicating the involvement of an autophagic mechanism that leads to lysosome-mediated destruction of the parasite. Estimation of the rate of lysosomal fusion with parasite-containing vacuoles using BSA-gold labeling showed that IGTP deficiency decreased the frequency of such events by $\sim 2-10-$ fold (Fig. $4 \mathrm{H}$ ). complex to host cytosol. Severed plasmalemma can fold onto itself, forming membrane double layers or whorls (J). (K) Detached parasite plasma membrane forming complex stacks. Note layers of detached plasmalemma held together by electron-dense bridging structures magnified in inset. T.g., T. gondii; black arrows, PVM; white arrows, PV-associated ER; black arrow heads, T. gondii plasma membrane; white arrow heads, T. gondii inner membrane complex; bars, $500 \mathrm{~nm}$. 
A

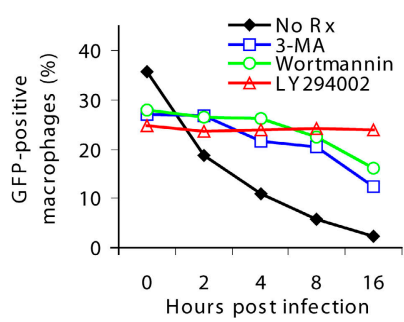

B

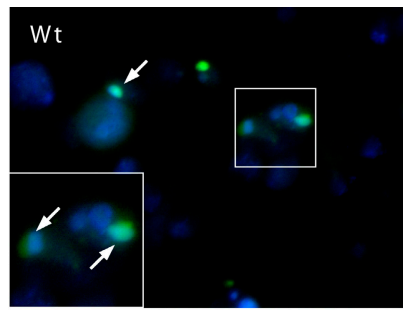

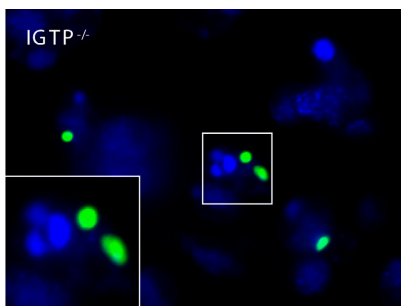

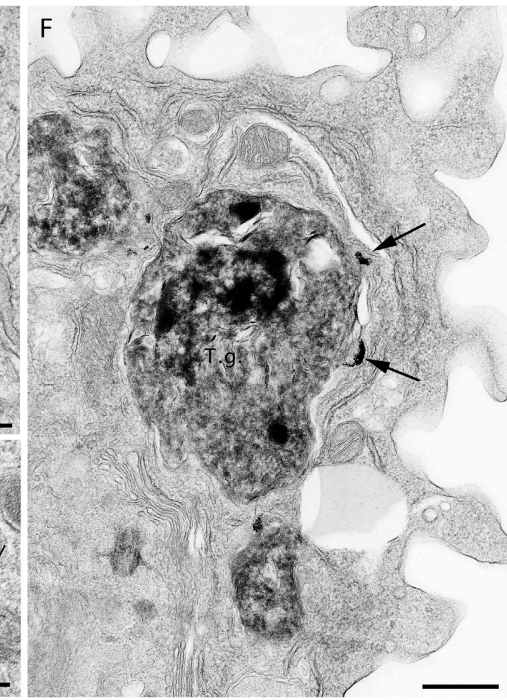

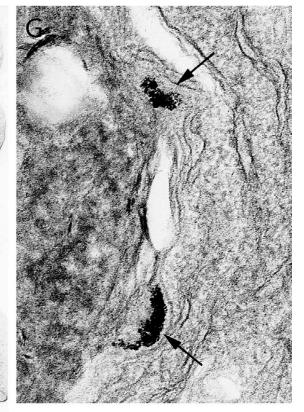

$\mathrm{H}$

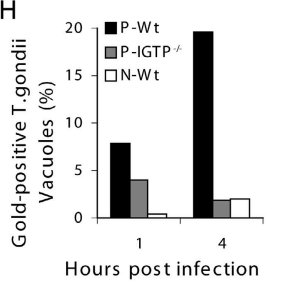

D

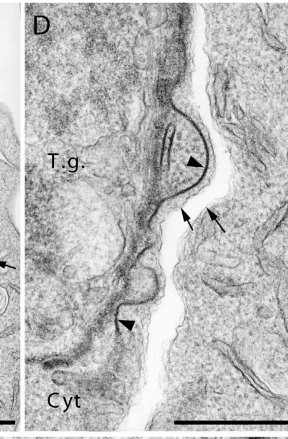

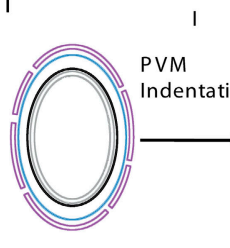

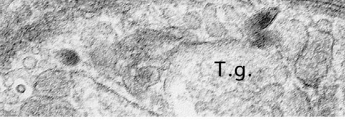

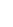

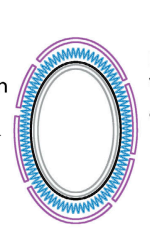

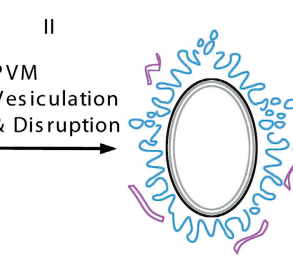

III

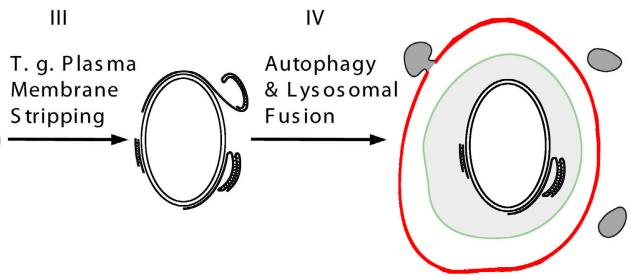

Figure 4. Autophagic elimination of $T$. gondii in primed macrophages. (A) PI3K inhibitors (100 nM wortmannin, $100 \mu \mathrm{M}$ LY294002, and 10 mM 3-MA 30 min before and after GFP-PTG infection) attenuated rapid parasite elimination in primed WT macrophages. (B) Colocalization of GFP-PTG (green) with MDC (blue) staining in primed WT macrophages at $2 \mathrm{~h}$ after infection. ( $\mathrm{C}$ and $\mathrm{D}$ ) Autophagosome formation in primed WT macrophages. Arrows point to the autophagosome outer membrane. Note the space between the outer and inner membranes and the denuded T. gondii with part of host cytoplasm (Cyt) within autophagosomes. (D) Autophagosome formation around a partially denuded parasite.

The pleitropic effect of IGTP deficiency on the killing activity of primed macrophages raises the question of when IGTP participates in the steps of PVM indentation, vesiculation and disruption, and parasite plasma membrane stripping. The p47 GTPase, IIGP, has been localized by immunofluorescence microscopy to the PV in astrocytes (16), but whether IGTP is also found in the PV remains controversial (24). We therefore performed cryo- immunoelectron microscopy to visualize IGTP localization within primed and T. gondiiinfected WT macrophages. Using IGTP-deficient macrophages as negative controls, clustered IGTP-specific labeling
Note detachment and folding of the electron-dense plasmalemma (arrow heads). (E) Fusion of flattened vesicles (arrows) giving rise to double membrane autophagosomes. (F) Fusion of lysosomes with T. gondiicontaining autophagic vacuoles. Arrows point to lysosomes containing 6-nm gold particles. (G) High magnification of the fusion event shown in F. (H) EM quantification of lysosomal fusion with T. gondii. (I) Proposed phases of T. gondii elimination in primed macrophages. Black, T. gondii plasmalemma; gray, T. gondii inner membrane complex; blue, PVM; magenta, ER; green, autophagosome inner membrane; red, autophagosome outer membrane; gray vesicles, lysosomes; bars, $500 \mathrm{~nm}$.

adjacent to the parasite was observed (Fig. 5 A), showing that IGTP is positioned to take part in some of the abovementioned membrane-disruptive events. Strikingly, IGTP immunostaining was also focally associated with autophagosome-like structures containing denuded T. gondii (Fig. 5 B), suggesting IGTP's role in autophagosome formation or maintenance (25).

Unlike Mycobacteria and Salmonella, which establish vacuolar niches along the endo-lysosomal route, apicomplexan parasites present special challenges to effector cells in their apparent seclusion from lysosome fusion. The series of events 

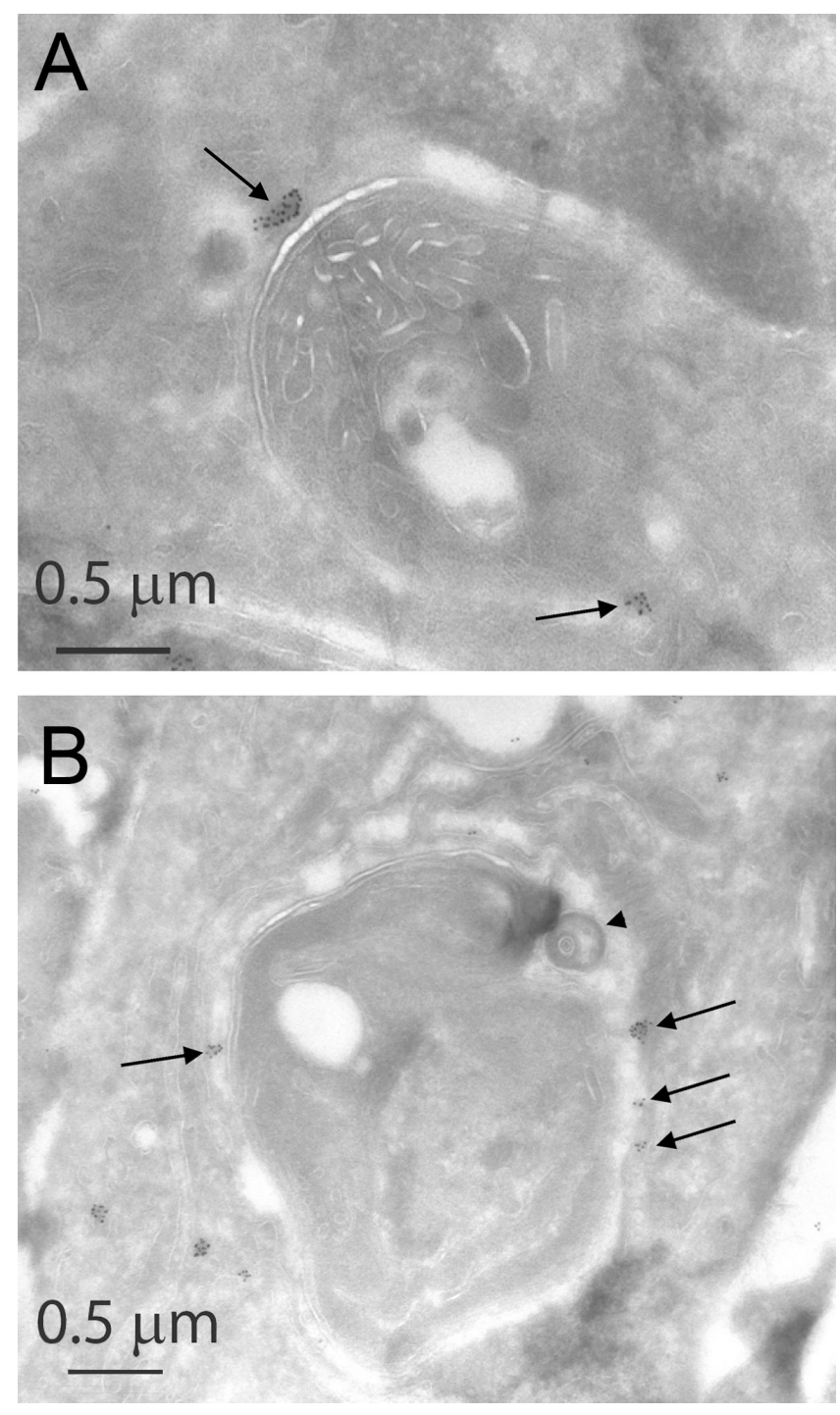

Figure 5. Localization of IGTP in T. gondii-infected primed WT macrophages. (A) IGTP immunoreactivity (15-nm gold, highlighted by arrows) in clusters adjacent to parasite (T. gondii). (B) IGTP immunoreactivity (highlighted by arrows) associated with the periphery of an autophagosome-like profile, with the characteristic circumferential clearance around the denuded parasite (T. gondii). Arrow head pinpoints stripped parasite plasmalemma, similar to one shown in Fig. 4 C.

we have described here outline a previously unrecognized and mechanistically distinct solution to the problem posed by nonfusogenic intracellular pathogens (Fig. 4 I). It remains unclear why autophagosome formation around $T$. gondii and lysosome fusion with the parasite were not observed in residual parasites after PVM disruption in astrocytes (16). This disparity may arise simply from methodological differences (in vitro activation of cultured astrocytes vs. in vivo priming of inflammatory macrophages) or may be a true reflection of innate differences in the defense arsenal of parenchymal (astrocyte) versus professional effector cells (macrophages). A related question yet to be resolved is whether the autopha- golysosomal degradation of denuded T. gondii (phase IV) is the direct cause of parasite demise or it simply represents a cell-type-restricted cytosolic response to clear $T$. gondii remnants after parasite killing in the preceding steps (phase I, II, or III). It will be interesting to find out whether other apicomplexan parasites such as Cryptosporidum, Eimeria, and Plasmodium are subject to the same series of PVM disruption and pathogen membrane denudation reactions after invasion of immune-activated intestinal and hepatic cells, respectively. Another important question is whether human cells can execute the same aggressive parasite elimination strategies described here using compensatory mechanisms given the apparent loss of the entire family of IFN- $\gamma$-responsive p47 GTPases genes during primate evolution (26).

\section{MATERIALS AND METHODS \\ Experimental animals}

C57BL/6, iNOS ${ }^{-1-}$, Sjl (CD45 congenic), and $\mu \mathrm{Mt}$ mice were obtained from The Jackson Laboratory. These mice, along with $\mathrm{IGTP}^{-/-}$and $\mathrm{IGTP}^{-/-} \mathrm{NOS}^{-/-}$mice, were bred and maintained under specific pathogenfree conditions at the Brown University animal care facility. Mice of both sexes between 5 and $12 \mathrm{wk}$ old were used for experiments. Studies were performed in accordance with guidelines of the Brown University Institutional Animal Care and Use Committee.

\section{Parasites}

The uracil auxotrophic CPS strain of T. gondii was provided by D. Bzik (Dartmouth Medical School, Hanover, NH; reference 10). GFP-PTG was obtained from American Type Culture Collection (no. 50941; reference 27). CPS parasites were $\gamma$ irradiated at 15,000 rads before use. In some experiments, fresh parasites fixed with $3 \%$ paraformaldehyde were used.

\section{In vivo priming}

For priming, $10^{6}$ irradiated CPS were injected i.p. At day 4 after initial injection, another dosage of the same parasites $\left(10^{5}\right.$, i.p.) was introduced to boost the priming effect. Primed mice were used on day 7 .

\section{In vivo infection and survival}

GFP-PTG resuspended in PBS was injected into the peritoneal cavity of primed mice. At different time points after infection, PECs were harvested by lavaging with RPMI medium containing 5\% FBS (Hyclone). Flow cytometry was used to monitor the infection rate in PECs. In survival experiments, primed WT and $\mathrm{IGTP}^{-/-}$mice were challenged with $2.5 \times 10^{5}$ GFP-PTG and mice were monitored daily.

\section{Ex vivo infection and treatments}

PECs from primed or thioglycolate-injected mice were mixed with GFP-PTG in 1-2 $\mathrm{ml}$ of invasion medium (DMEM with $1 \% \mathrm{FBS}$, multiplicity of infection [MOI] of $0.5-1)$ in $15-\mathrm{ml}$ conical tubes for $15-30 \mathrm{~min}$ at $37^{\circ} \mathrm{C}$. To terminate infection, cold RPMI with 5\% FBS was added to fill the tubes. Free parasites were removed by differential centrifugation. Infected PECs were resuspended in RPMI with 10\% FBS and aliquoted for assays at different time points.

In some experiments, PECs from primed Sjl $\left(\mathrm{CD} 45.1^{+}\right)$and $\mathrm{IGTP}^{-/-}$ $\left(\mathrm{CD} 45.2^{+}\right.$) were mixed together before GFP-PTG infection (MOI of 1, 15 min) ex vivo. Surface staining for CD45.1 and CD45.2 antigen was used to differentiate the two cell populations by flow cytometry.

IFN- $\boldsymbol{\gamma}$ treatment. In experiments where activation of thioglycolate-elicited peritoneal macrophages in vitro was desired, IFN- $\gamma(1 \mathrm{ng} / \mathrm{ml})$ treatment started $12 \mathrm{~h}$ before infection and was maintained after infection.

Bafilomycin-A1 treatment. To inhibit lysosome function, PECs were pretreated with $100 \mathrm{nM}$ bafilomycin-A1 for $1 \mathrm{~h}$. After infection with 
GFP-PTG, bafilomycin-A1 was resupplied and maintained throughout the experiment.

PI3K inhibition. $100 \mathrm{nM}$ wortmannin, $100 \mu \mathrm{M}$ LY294002, and $10 \mathrm{mM}$ 3-methyladenine were used to inhibit PI3K activity. PI3k inhibitor treatment started 30 min before infection and was maintained after infection. All PECs were maintained and all reagents were diluted in RPMI with 10\% FBS unless otherwise stated.

\section{Flow cytometry}

PECs were fixed with CytoFix (BD Biosciences) before analysis. GFP positivity detected by flow cytometry (FACSCalibur; Becton Dickinson) was used to determine the infection rate in PECs. Shown in this study are infection rates in macrophages, defined as being forward scatter ${ }^{\text {high }}$ and side scatter high. These large, granular cells were $>90 \% \mathrm{CD} 11 \mathrm{~b}^{+} \mathrm{F} 4 / 80^{+}$, as determined in a separate experiment with surface staining for CD 11b (BD Biosciences) and F4/80 (eBioscience) antigens.

\section{Parasite viability assay}

The viability of $T$. gondii residing in host cells at different time points after infection was determined by plaque assay. In brief, a small volume $(0.5 \mathrm{ml})$ of infected PEC suspension was passed through a 27-gauge needle 10 times to liberate the parasites. The needle-passaged lysates (volume adjusted to ensure equal plating) were plated onto human foreskin fibroblast monolayers in 24-well plates. $6 \mathrm{~d}$ after plating, the number of plaques was counted and the change (relative to time 0 ) in plaque-forming efficiency was calculated.

\section{Fluorescent staining and microscopy}

LAMP1 staining. PECs infected with GFP-PTG in vivo were seeded onto FBS-coated coverslips in 24-well plates for $2 \mathrm{~h}$ and washed with PBS before being prepared for LAMP1 staining. For ex vivo infection, PECs were allowed to adhere onto coverslips for $3 \mathrm{~h}$, and nonadherent cells were washed away with PBS. After a 10-min incubation with GFP-PTG in invasion medium at $37^{\circ} \mathrm{C}$, adherent PECs were washed extensively with PBS to remove extracellular parasites. Infected cells were incubated in RPMI with $10 \%$ medium at $37^{\circ} \mathrm{C}$ until being processed at different time points for immunofluorescent staining.

Adherent PECs were washed with PBS twice before fixation with 3\% paraformaldehyde for $15 \mathrm{~min}$ at room temperature. After permeabilization (0.1\% Triton X-100 in PBS for $10 \mathrm{~min}$ ) and blocking (5\% goat serum, 1\% BSA, $0.05 \%$ Tween 20 in PBS for $1 \mathrm{~h}$ ), cells were incubated with LAMP1 antibody (1D4B; $0.335 \mathrm{ug} / \mathrm{ml}$, diluted in blocking buffer; DSHB, University of Iowa) for $45 \mathrm{~min}$. Rat IgG was included as negative control. After washing, cells were further incubated with Alexa Fluor 568-conjugated goat antirat antibody (1:1,000, diluted in blocking buffer; Invitrogen) for $30 \mathrm{~min}$, followed by washing and DAPI staining. Stained coverslips were mounted onto slides with ProLong antifade (Invitrogen) and sealed with nail polish.

IGTP staining. PECs from day 7 primed mice were harvested and allowed to adhere onto coverslips for $4 \mathrm{~h}$ before being fixed and stained according to the similar procedure described above. $1 \mathrm{ug} / \mathrm{ml}$ monoclonal anti-IGTP antibody (BD Biosciences) conjugated with Alexa Fluor 647 (Invitrogen) was used for staining.

Lysosome labeling with Texas red dextran. Adherent PECs were pulsed with lysine-fixable dextran Texas red $(0.5 \mathrm{mg} / \mathrm{ml}, 10 \mathrm{kD}$; Invitrogen $)$ for $1 \mathrm{~h}$. After washing and $3 \mathrm{~h}$ of incubation, dextran was chased into lysosomes before GFP-PTG infection (28).

MDC staining. At $2 \mathrm{~h}$ after GFP-PTG infection, adherent PECs were incubated with MDC (0.05 mM in PBS; Sigma-Aldrich) for $10 \mathrm{~min}$. After three washes, cells were left in PBS and immediately photographed.

Fluorescent photomicrographs were acquired using a Zeiss Axiovert 200M microscope. Confocal images were acquired using a Leica TCS SP2 AOBS spectral confocal microscope.

\section{EM}

Before GFP-PTG infection, naive and primed macrophages were pulsed with $6 \mathrm{~nm}$ BSA-gold and incubated for $3 \mathrm{~h}$ to chase this label into lysosomes. $15 \mathrm{~nm}$ BSA-gold was present during the 15-min pulse infection to distinguish between actively invaded and phagocytosed T. gondii parasites. Samples were prepared for transmission EM analysis at 1, 2, and $4 \mathrm{~h}$ after infection as described previously (29). For immunoelectron microscopy, cells were fixed in $4 \%$ paraformaldehyde (Electron Microscopy Sciences) in $0.25 \mathrm{M}$ Hepes, ph 7.4, for $1 \mathrm{~h}$ at room temperature, and then in $8 \%$ paraformaldehyde in the same buffer overnight at $4^{\circ} \mathrm{C}(30)$. They were infiltrated, frozen, and sectioned as previously described (30). The sections were immunolabeled with anti-IGTP antibody (1:20 in PBS/1\% fish skin gelatin), and then with anti-mouse IgG antibodies, followed directly by $15 \mathrm{~nm}$ of protein Agold particles (Department of Cell Biology, Medical School, Utrecht University) before examination with a Philips CM120 electron microscope (Eindhoven) under $80 \mathrm{kV}$.

\section{Online supplemental material}

Fig. S1 shows the scheme for the CPS-priming GFP-PTG challenge experimental system. Fig. S2 shows the plaque assay determination of T. gondii viability after ex vivo infection of peritoneal cells. Fig. S3 shows the cellautonomous effect of IGTP on parasite clearance by primed PECs. Fig. S4 demonstrates that rapid parasite elimination does not require B cells and Ig. Confocal photomicrographs of dextran-labeled macrophages infected with GFP-PTG are shown in Fig. S5. Fig. S6 shows electron micrographs of parasite degeneration in primed WT, but not in IGTP knockout, macrophages. Fig. S7 shows PVM indentation in a primed WT macrophage. Fig. S8 shows that the detached parasite plasma membrane can form complex stacks. Fig. S9 shows additional examples of autophagosomes containing denuded parasites. Fig. S10 is an electron micrograph of T. gondii remnants within autophagolysosomes containing flocculated 15-nm gold particles used in this experiment to trace lysosomes. Supplementary videos of stacked images (Videos S1 and S2) are also provided. Online supplemental material is available at http://www.jem.org/cgi/content/full/jem.20061318/DC1.

We thank J. Nathanson and M. Belda for help with graphics, G. Williams, G. Ward, and J.F. Dubremetz for advice and discussion.

Financial support for this work was provided by National Institutes of Health (NIH) grant Al 50618 to G.S. Yap. G.A. Taylor was supported by NIH grant AI 57831 and a VA merit review grant.

The authors have no conflicting financial interests.

Submitted: 21 June 2006

Accepted: 3 August 2006

\section{REFERENCES}

1. Dubey, J.P., and C.P. Beattie. 1988. Toxoplasmosis of Animals and Man. CRC Press, Boca Raton. 220 pp.

2. Miller, L.H., D.I. Baruch, K. Marsh, and O.K. Doumbo. 2002. The pathogenic basis of malaria. Nature. 415:673-679.

3. Sinai, A.P., and K.A. Joiner. 1997. Safe haven: the cell biology of nonfusogenic pathogen vacuoles. Annu. Rev. Microbiol. 51:415-462.

4. Jones, T.C., and J.G. Hirsch. 1972. The interaction between Toxoplasma gondii and mammalian cells. II. The absence of lysosomal fusion with phagocytic vacuoles containing living parasites. J. Exp. Med. 136:1173-1194.

5. Schwab, J.C., C.J. Beckers, and K.A. Joiner. 1994. The parasitophorous vacuole membrane surrounding intracellular Toxoplasma gondii functions as a molecular sieve. Proc. Natl. Acad. Sci. USA. 91:509-513.

6. Sinai, A.P., P. Webster, and K.A. Joiner. 1997. Association of host cell endoplasmic reticulum and mitochondria with the Toxoplasma gondii parasitophorous vacuole membrane: a high affinity interaction. J. Cell Sci. 110:2117-2128.

7. Yap, G.S., and A. Sher. 1999. Effector cells of both nonhemopoietic and hemopoietic origin are required for interferon (IFN) $-\gamma-$ and tumor necrosis factor (TNF)- $\alpha$-dependent host resistance to the intracellular pathogen, Toxoplasma gondii. J. Exp. Med. 189:1083-1092. 
8. Adams, L.B., J.B. Hibbs Jr., R.R. Taintor, and J.L. Krahenbuhl. 1990. Microbiostatic effect of murine-activated macrophages for Toxoplasma gondii. Role for synthesis of inorganic nitrogen oxides from L-arginine. J. Immunol. 144:2725-2729.

9. Pfefferkorn, E.R. 1984. Interferon gamma blocks the growth of Toxoplasma gondii in human fibroblasts by inducing the host cells to degrade tryptophan. Proc. Natl. Acad. Sci. USA. 81:908-912.

10. Fox, B.A., and D.J. Bzik. 2002. De novo pyrimidine biosynthesis is required for virulence of Toxoplasma gondii. Nature. 415:926-929.

11. Remington, J.S., J.L. Krahenbuhl, and J.W. Mendenhall. 1972. A role for activated macrophages in resistance to infection with Toxoplasma. Infect. Immun. 6:829-834.

12. Taylor, G.A., C.M. Collazo, G.S. Yap, K. Nguyen, T.A. Gregorio, L.S. Taylor, B. Eagleson, L. Secrest, E.A. Southon, S.W. Reid, et al 2000. Pathogen-specific loss of host resistance in mice lacking the IFNgamma-inducible gene IGTP. Proc. Natl. Acad. Sci. USA. 97:751-755.

13. Scharton-Kersten, T.M., G. Yap, J. Magram, and A. Sher. 1997. Inducible nitric oxide is essential for host control of persistent but not acute infection with the intracellular pathogen Toxoplasma gondii.J. Exp. Med. 185:1261-1273.

14. Mordue, D.G., S. Hakansson, I. Niesman, and L.D. Sibley. 1999. Toxoplasma gondii resides in a vacuole that avoids fusion with host cell endocytic and exocytic vesicular trafficking pathways. Exp. Parasitol. 92:87-99.

15. Haupts, U., S. Maiti, P. Schwille, and W.W. Webb. 1998. Dynamics of fluorescence fluctuations in green fluorescent protein observed by fluorescence correlation spectroscopy. Proc. Natl. Acad. Sci. USA. 95:13573-13578.

16. Martens, S., I. Parvanova, J. Zerrahn, G. Griffiths, G. Schell, G. Reichmann, and J.C. Howard. 2005. Disruption of Toxoplasma gondii parasitophorous vacuoles by the mouse p47-resistance GTPases. PLoS Pathog. 1:e24.

17. Shintani, T., and D.J. Klionsky. 2004. Autophagy in health and disease: a double-edged sword. Science. 306:990-995.

18. Nakagawa, I., A. Amano, N. Mizushima, A. Yamamoto, H. Yamaguchi, T. Kamimoto, A. Nara, J. Funao, M. Nakata, K. Tsuda, et al. 2004 Autophagy defends cells against invading group A Streptococcus. Science. 306:1037-1040

19. Ogawa, M., T. Yoshimori, T. Suzuki, H. Sagara, N. Mizushima, and C. Sasakawa. 2005. Escape of intracellular Shigella from autophagy. Science. 307:727-731.
20. Kirkegaard, K., M.P. Taylor, and W.T. Jackson. 2004. Cellular autophagy: surrender, avoidance and subversion by microorganisms. Nat. Rev. Microbiol. 2:301-314.

21. Petiot, A., E. Ogier-Denis, E.F. Blommaart, A.J. Meijer, and P. Codogno. 2000. Distinct classes of phosphatidylinositol 3'-kinases are involved in signaling pathways that control macroautophagy in HT-29 cells. J. Biol. Chem. 275:992-998.

22. Munafo, D.B., and M.I. Colombo. 2001. A novel assay to study autophagy: regulation of autophagosome vacuole size by amino acid deprivation. J. Cell Sci. 114:3619-3629.

23. Dunn, W.A., Jr. 1990. Studies on the mechanisms of autophagy: formation of the autophagic vacuole. J. Cell Biol. 110:1923-1933.

24. Butcher, B.A., R.I. Greene, S.C. Henry, K.L. Annecharico, J.B Weinberg, E.Y. Denkers, A. Sher, and G.A. Taylor. 2005. p47 GTPases regulate Toxoplasma gondii survival in activated macrophages. Infect. Immun. 73:3278-3286.

25. Gutierrez, M.G., S.S. Master, S.B. Singh, G.A. Taylor, M.I. Colombo, and V. Deretic. 2004. Autophagy is a defense mechanism inhibiting BCG and Mycobacterium tuberculosis survival in infected macrophages. Cell. 119:753-766.

26. Bekpen, C., J.P. Hunn, C. Rohde, I. Parvanova, L. Guethlein, D.M. Dunn, E. Glowalla, M. Leptin, and J.C. Howard. 2005. The interferoninducible p47 (IRG) GTPases in vertebrates: loss of the cell autonomous resistance mechanism in the human lineage. Genome Biol. 6:R92.

27. Kim, K., M.S. Eaton, W. Schubert, S. Wu, and J. Tang. 2001 Optimized expression of green fluorescent protein in Toxoplasma gondii using thermostable green fluorescent protein mutants. Mol. Biochem. Parasitol. 113:309-313.

28. Roy, D., D.R. Liston, V.J. Idone, A. Di, D.J. Nelson, C. Pujol, J.B. Bliska, S. Chakrabarti, and N.W. Andrews. 2004. A process for controlling intracellular bacterial infections induced by membrane injury. Science. 304:1515-1518.

29. Ferguson, D.J., F.L. Henriquez, M.J. Kirisits, S.P. Muench, S.T. Prigge, D.W. Rice, C.W. Roberts, and R.L. McLeod. 2005. Maternal inheritance and stage-specific variation of the apicoplast in Toxoplasma gondii during development in the intermediate and definitive host. Eukaryot. Cell. 4:814-826.

30. Folsch, H., M. Pypaert, P. Schu, and I. Mellman. 2001. Distribution and function of AP-1 clathrin adaptor complexes in polarized epithelial cells. J. Cell Biol. 152:595-606. 